\title{
Perfil Cognitivo e Motor de Crianças Nascidas Prematuras em Idade Escolar: Revisão de Literatura
}

\author{
Motor and Cognitive Profile of Premature Children at School Age: Review of Literature
}

\section{Débora ZAP do Nascimento ${ }^{1}$, Kátia de PP Carvalho ${ }^{1}$, Cristina Iwabe ${ }^{2}$}

\section{RESUMO}

Objetivo. Descrever o perfil cognitivo e motor de crianças nascidas prematuras em idade escolar, a fim de detalhar o seu prognóstico clínico. Método. Para este estudo foi realizada uma revisão bibliográfica por meio de pesquisa no banco de dados Lilacs, EMBASE, Cochrane, Scielo, Medline, Pubmed e bibliotecas virtuais de teses e dissertações da Universidade de São Paulo - USP e Universidade Estadual de Campinas - UNICAMP, compreendendo artigos científicos dos últimos 10 anos. Como critérios de inclusão foram considerados aqueles com relevância acadêmica científica e de acordo com o objetivo proposto em nosso estudo. Foram excluídos aqueles sem relevância acadêmica, e fora do contexto do objetivo citado. Resultados. Foram encontrados 30 artigos científicos, excluindo 17 que não se enquadravam no objetivo proposto, totalizando 13 estudos. Observou-se que as crianças pré-termo, quando comparadas com aquelas a termo, apresentam relevantes índices de atraso no desenvolvimento cognitivo e motor, colaborando para o insucesso escolar. Conclusão. Crianças nascidas prematuramente apresentam menores escores tanto nas tarefas cognitivas quanto motoras quando comparadas com crianças nascidas a termo.

Unitermos. Prematuridade, Desenvolvimento Infantil, Idade Escolar, Cognição.

Citaçáo. Nascimento DZAP, Carvalho KPP, Iwabe C. Perfil Cognitivo e Motor de Crianças Nascidas Prematuras em Idade Escolar: Revisão de Literatura.

\begin{abstract}
Objective. Describe the cognitive and motor profile in premature children at school age in order to detail their clinical prognosis. Method. A bibliography review was realized by search on scientific articles on database of Lilacs, EMBASE, Cochrane, Scielo, Medline, Pubmed and researches in virtual library at São Paulo University USP and Campinas State University - UNICAMP Scielo, Medline and Pubmed sites from the last 10 years. It was considered scientific and academic relevance according to the objective proposed in our study. It was excluded those with no academic relevance, and outside the context of the objective cited. Results. It was found 30 scientific articles, excluding 17 who didn't meet the proposed objective, with a total of 13 studies. It was observed that premature children when compared with term children, present relevant delayed cognitive and motor development, contributing to academic failure. Conclusion. Premature children show lower scores on cognitive and motor tasks as compared to children born at term.
\end{abstract}

Keywords. Prematurity, Child Development, School Age, Cognition.

Citation. Nascimento DZAP, Carvalho KPP, Iwabe C. Motor and Cognitive Profile of Premature Children at School Age: Review of Literature.
Trabalho realizado como parte da conclusão do Curso de Fisioterapia do Centro Universitário Padre Anchieta - Jundiaí-SP, Brasil.

1.Graduadas do Curso de Fisioterapia - Centro Universitário Padre Anchieta, Jundiaí-SP, Brasil.

2.Fisioterapeuta, Doutora em Ciências Médicas UNICAMP; Docente do Curso de Fisioterapia Centro Universitário Padre Anchieta, Jundiaí-SP, Brasil.
Endereço para correspondência: Cristina Iwabe Rua Mata dos Pinhais, 61 casa 76 CEP 13082-761, Campinas-SP, Brasil. E-mail: crisiwabe@hotmail.com; ciwabe@fcm.unicamp.br 


\section{INTRODUÇÃO}

O termo prematuro ou pré-termo refere-se a crianças nascidas com menos de 37 semanas gestacionais. Atualmente, a perspectiva de vida de uma criança nascida prematuramente tem aumentado gradativamente devido aos grandes avanços tecnológicos advindos da assistência obstétrica e neonatal oferecida ${ }^{1,2}$.

A etiologia do nascimento pré-termo não é multifatorial. Diversos fatores de risco clássicos como infecçôes, partos múltiplos, hipertensão induzida pela gravidez, trabalho extenuante, baixo índice de massa corpórea, de peso insuficiente na gravidez, reprodução assistida, colo uterino curto, intervalo interpartal curto, baixa escolaridade, raça negra e história anterior de nascimento pré-termo tem sido responsabilizados por apenas um terço dos partos prematuros ${ }^{3,4}$.

Apesar do constante avanço dos estudos relacionados à prematuridade, tal condição pode revelar-se desfavorável para o desenvolvimento da criança. Prejuízos relacionados ao desenvolvimento físico, cognitivo, emocional, e comportamental são encontrados frequentemente nessa população. Considerando-se que o desenvolvimento global da criança envolve o desempenho das funçôes visuoperceptiva, motora, atencionais, e as manifestações de comportamento e emoções, que são resultados da integridade estrutural e funcional das estruturas neurológicas, não são surpreendentes a ocorrência das alteraçóes anteriormente citadas no caso de crianças prematuras, já que nascem neurologicamente mais imaturas do que a termo, e com taxas de morbidade perinatal ${ }^{5,6}$.

No entanto, após a década de 90, com os avanços que produziram maiores índices de sobrevivência para recém-nascidos prematuros, o reconhecimento precoce de déficits cognitivos e comportamentais nessa população com maiores riscos de adversidades durante o surgimento de longo prazo se tornou significativo em termos de qualidade de vida futura ${ }^{5,6}$.

O desenvolvimento motor é considerado como um processo sequencial contínuo, relacionado à idade cronológica, pelo qual o ser humano adquire uma enorme quantidade de habilidades motoras, as quais progridem do movimento simples e desorganizado para a execução de habilidades motoras organizadas e complexas ${ }^{7}$.

O desenvolvimento motor atípico não se vincula, obrigatoriamente, a presença de alteraçóes neurológicas ou estruturais. Mesmo crianças que não apresentam sequelas graves podem apresentar comprometimento em algumas áreas de seu desenvolvimento neuropsicomotor ${ }^{7}$.

A cognição constitui-se em variável importante na estruturaçáo e dinâmica do desenvolvimento global da criança e pode fornecer indicadores sugestivos acerca do seu desempenho futuro ${ }^{8}$.

Crianças prematuras e com baixo peso podem apresentar desvantagens no âmbito escolar com relação à inteligência, ao desempenho escolar e às dificuldades comportamentais quando comparadas com crianças nascidas a termo e com peso normal. Prematuros nascidos com baixo peso podem evidenciar prejuízos neurocomportamentais como déficits cognitivos, dificuldades de linguagem, anormalidades neurológicas, atrasos no desenvolvimento e maior risco de sequelas, como surdez, cegueira, convulsóes e paralisia cerebral ${ }^{9}$.

Desse modo o objetivo deste estudo foi descrever o perfil cognitivo e motor de crianças nascidas prematuras em idade escolar, a fim de detalhar o seu prognóstico clínico.

\section{MÉTODO}

Para este estudo foi realizada uma revisão bibliográfica por meio de pesquisa no banco de dados Lilacs, EMBASE, Cochrane, Scielo, Medline, Pubmed e bibliotecas virtuais de teses e dissertações da Universidade de São Paulo - USP e Universidade Estadual de Campinas - UNICAMP. A pesquisa englobou artigos publicados entre 2001 e 2010, nos idioma português e inglês, encontrados por pesquisa direta ou por referências de outros artigos consultados.

Foram utilizadas as seguintes palavras-chaves: prematuridade (prematurity), desenvolvimento infantil (child development), idade escolar (school age) e cognição (cognition). A pesquisa foi realizada em bibliotecas virtuais, banco de dados e biblioteca da Faculdade Padre Anchieta.

Como critério de inclusão considerou-se os artigos científicos de estudos controlados ou revisão, indexados em revistas científicas com critérios de qualificação pela Capes - Qualis- A e B, englobando uma população de crianças em idade escolar de 3 a 6 anos de idade. Além 
disso, foram incluídos livros acadêmicos que estavam de acordo com os objetivos propostos no nosso estudo, Foram excluídos aqueles com qualificação pela Capes abaixo de $\mathrm{C}$, e fora do contexto dos objetivos citados.

\section{RESULTADOS}

Foram encontrados 30 artigos científicos de acordo com as palavras-chaves utilizadas (desenvolvimento motor e cognitivo, prematuridade e idade escolar). Foram excluídas 17 referências, pois não associavam a prematuridade com o desenvolvimento motor e cognitivo em idade escolar.

12 artigos selecionados eram estudos clínicos controlados, e quatro eram revisóes bibliográficas. Dentre as pesquisas analisadas, dois relatavam atrasos motores, principalmente na coordenação motora, em crianças nascidas prematuras em idade escolar ${ }^{10-15}$. Os demais enfatizaram a limitação nas funçóes cognitivas, comportamentais e de linguagem na população estudada ${ }^{6,9,11-14,16-23}$.

$\mathrm{Na}$ Tabela 1 encontram-se a relação dos artigos selecionados, com a descrição dos perfis cognitivos e motores das crianças nascidas prematuras em idade escolar.

\section{DISCUSSÃo}

Os resultados deste estudo indicam um aumento significativo do risco de atraso no desenvolvimento cognitivo e motor. A idade gestacional e o peso ao nascimento representam fatores preditivos importantes no prognóstico do desenvolvimento infantil. Apesar da apresentação inicial saudável, pais, médicos, especialistas em desenvolvimento infantil e profissional da educação, precisam estar cientes dos riscos de insucesso escolar e problemas comportamentais, para que a solicitação para serviços de intervenção precoce seja feita ${ }^{8,12,17,24,25}$.

Diversas funçôes motoras, executivas ou sensoriais podem estar deficientes na criança prematura como, déficits relacionados ao quociente de inteligência, memória, capacidade para cálculos, função cognitiva global, desenvolvimento motor, função motora grossa, aprendizado, linguagem, habilidades viso-motor, planejamento, pensamento racional, associativo e atenção, podendo ter consequência em sua educação e qualidade de vida futu$\mathrm{ra}^{6,9,19,22}$.

A literatura refere que neonatos nascidos com bai- xo peso são considerados de risco para alteraçóes globais tanto receptivas quanto expressiva, especialmente durante os anos pré-escolares e escolares ${ }^{26,27}$.

Durante o período escolar observou-se menor desempenho nas atividades de aritmética, capacidade gráfica e de linguagem, tanto expressiva como compreensiva, na aprendizagem da leitura, escrita ou soletração, reforçando a necessidade de se prover estimulação adequada à criança, envolvendo a família e a escola, já que apresentam maior taxa de repetência escolar ${ }^{9,28-30}$.

Crianças prematuras apresentaram uma prevalência duas vezes maior de desatenção, hiperatividade, problemas emocionais, comportamentais e de relacionamento com colegas, além de despenderem mais tempo para realizar as atividades propostas o que pode ter um impacto funcional, resultando em lentidão na escrita. Observou-se também atraso no desenvolvimento motor global (motricidade fina, grossa e viso-motor), evidentes na idade pré-escolar, quando se consolidam as habilidades motoras ${ }^{12,13,15,17,31}$.

Crianças prematuras podem apresentar alteraçóes concomitantes de deficiências cognitivas, com déficit motor comportamental principalmente em idade escolar ou mais tardiamente. Tais problemas cognitivos e educacionais podem estar relacionados às anomalias cerebrais $^{10,16,32,33}$.

Estudos recentes mostram a preocupação com o desenvolvimento intelectual deste grupo de crianças, já que a deficiência no desenvolvimento cognitivo pode interferir significativamente em seu rendimento escolar. Desse modo, a estimulaçáo cognitiva dentro do ambiente familiar é essencial para a obtenção de bons resultados em seu crescimento e desenvolvimento ${ }^{25,34,35}$.

Os prematuros devem ser analisados criteriosamente, tanto no aspecto cognitivo, comportamental ou motor. Vários fatores contribuem para o futuro desempenho dessas crianças, o que exige uma observação de suas habilidades a partir de uma abordagem dinâmica, onde diferentes situaçóes podem vir a trazer interferências em momentos específicos do desenvolvimento, comprometendo futuras aquisiçóes ${ }^{30,36}$.

Apesar de a literatura internacional ser rica em trabalhos sobre o acompanhamento e estimulação do desenvolvimento infantil ${ }^{6,9,10-12,14-16,18,20-22,24,35-41}$, as pesquisas 
Tabela 1

Resumo dos Artigos Selecionados

\begin{tabular}{|c|c|c|c|c|}
\hline AUTOR & ARTIGO & OBJETIVOS & POPULAÇÃO & CONCLUSĀO \\
\hline Benjamin et al. ${ }^{10}$ & $\begin{array}{l}\text { Early School-Age outcomes } \\
\text { of Late Preterm Infants }\end{array}$ & $\begin{array}{l}\text { Comparar o desenvolvimento } \\
\text { motor entre crianças a termo e } \\
\text { pré-termo, frequentadoras de } \\
\text { creche e jardim de infância. }\end{array}$ & $\begin{array}{l}\text { IG } 37 \text { a } 41 \text { semanas e } 34 \\
\text { a } 36 \text { semanas. } \\
7152 \text { crianças pré termo } \\
\text { e } 152661 \text { a termo. }\end{array}$ & $\begin{array}{l}36 \% \text { maior de risco para atraso do } \\
\text { desenvolvimento ou deficiência entre } \\
\text { lactentes prematuros moderados em } \\
\text { comparação com aquelas nascidas a } \\
\text { termo. }\end{array}$ \\
\hline Muthanna et al. ${ }^{11}$ & $\begin{array}{l}\text { Pervasive Behavior Proble- } \\
\text { ms at } 6 \text { years of Age in a } \\
\text { Total-Population Sample } \\
\text { of Children Born at } \leq 25 \\
\text { weeks of Gestation }\end{array}$ & $\begin{array}{l}\text { Avaliar o perfil comportamental } \\
\text { de crianças extremamente pre- } \\
\text { maturas em relação ao grupo } \\
\text { controle (a termo) }\end{array}$ & $\begin{array}{l}\text { IG } \leq 25 \text { semanas. } \\
200 \text { crianças no grupo } \\
\text { experimental, e } 180 \text { no } \\
\text { grupo controle. }\end{array}$ & $\begin{array}{l}\text { Os problemas comportamentais, como } \\
\text { hiperatividade, má conduta, défcit de } \\
\text { atenção e cognição, são mais frequentes } \\
\text { em crianças pré-termo do que aquelas } \\
\text { nascidas a termo, principalmente no } \\
\text { gênero masculino. }\end{array}$ \\
\hline $\begin{array}{l}\text { Delobel-Ayoub, } \\
\text { et al. }{ }^{12}\end{array}$ & $\begin{array}{l}\text { Behavioral Problems and } \\
\text { Cognitive Performance at } \\
5 \text { years of age after very pre- } \\
\text { term Bith: The EPIPAGE } \\
\text { Study }\end{array}$ & $\begin{array}{l}\text { Comparar a frequência de } \\
\text { problemas comportamentais } \\
\text { em crianças muito prematuras e } \\
\text { a termo aos } 5 \text { anos de idade. }\end{array}$ & $\begin{array}{l}\text { IG } 33 \text { semanas e } 39 \text { a } 40 \\
\text { semanas. } \\
1102 \text { crianças pré-termo } \\
\text { e } 375 \text { a termo. }\end{array}$ & $\begin{array}{l}\text { Houve o dobro de prevalência de sinto- } \\
\text { mas emocionais, de relacionamentos e } \\
\text { comportamentais em comparaçáo com } \\
\text { crianças nascidas a termo, associados à } \\
\text { baixa performance cognitiva. }\end{array}$ \\
\hline Magalhães et al. ${ }^{13}$ & $\begin{array}{l}\text { Estudo comparativo sobre } \\
\text { o desempenho percentual e } \\
\text { motor na idade escolar em } \\
\text { crianças nascidas pré-termo } \\
\text { e a termo }\end{array}$ & $\begin{array}{l}\text { Comparar o desenvolvimento } \\
\text { perceptual motor na idade } \\
\text { escolar, entre crianças nascidas } \\
\text { pré-termo e a termo. }\end{array}$ & IG até 34 semanas & $\begin{array}{l}\text { Crianças nascidas pré-termo apre- } \\
\text { sentaram menores escores nas provas } \\
\text { de equilíbrio, menor tônus postural, } \\
\text { menor capacidade de atividades viso } \\
\text { motora. }\end{array}$ \\
\hline Hughes $^{14}$ & $\begin{array}{l}\text { Motor, cognitive, and beha- } \\
\text { vioral disorders in children } \\
\text { on very preterm }\end{array}$ & $\begin{array}{l}\text { Analisar a taxa de comprome- } \\
\text { timento motor associado ao } \\
\text { comportamento e deficiência } \\
\text { cognitiva em uma região. }\end{array}$ & $\begin{array}{l}\text { IG }<32 \text { semanas. } \\
151 \text { crianças pré-termo e } \\
210 \text { controle. }\end{array}$ & $\begin{array}{l}\text { A deficiência motora persiste em so- } \\
\text { breviventes de nascimento prematuro, } \\
\text { apesar das melhorias no atendimento } \\
\text { e nâo se limitam a crianças menores } \\
\text { ou pré-termo. Eles podem existir inde- } \\
\text { pendentemente do déficit cognitivo e } \\
\text { comportamental, embora muitas vezes } \\
\text { coexistam. }\end{array}$ \\
\hline $\begin{array}{l}\text { Sullivan e } \\
\text { McGrath }^{15}\end{array}$ & $\begin{array}{l}\text { Perinatal morbidity, mild } \\
\text { motor delay, and later } \\
\text { school outcomes }\end{array}$ & $\begin{array}{l}\text { Investigar quatro dimensóes } \\
\text { do desempenho motor (motor } \\
\text { completo, motor fino, motor } \\
\text { grosso e integraçáo visual } \\
\text { motora). }\end{array}$ & $\begin{array}{l}\text { IG } \geq \text { e } \leq 37 \text { semanas } \\
134 \text { crianças saudáveis } \\
\text { a termo; } 134 \text { crianças } \\
\text { pré-termo ( } 41 \text { saudáveis, } \\
59 \text { com doença, } 34 \text { com } \\
\text { déficit neurológico). }\end{array}$ & $\begin{array}{l}\text { Os prematuros têm um risco três } \\
\text { vezes maior para o baixo desempenho } \\
\text { escolar, nas atividades motoras grossas } \\
\text { e finas. }\end{array}$ \\
\hline Anderson et al. ${ }^{16}$ & $\begin{array}{l}\text { Executive Functioning in } \\
\text { school-Aged Children Who } \\
\text { were Born very preterm } \\
\text { with extremely low birth } \\
\text { weight in the } 1990 \text { s. }\end{array}$ & $\begin{array}{l}\text { Analisar o perfil das funçôes } \\
\text { executivas em crianças de } 8 \\
\text { anos de idade, nascidas prema- } \\
\text { turas e de muito baixo peso }\end{array}$ & $\begin{array}{l}\mathrm{IG}<28 \text { semanas }(298 \\
\text { crianças pré-termo e } 262 \\
\text { controle })\end{array}$ & $\begin{array}{l}\text { Crianças prematuras, comparadas com } \\
\text { grupo controle (peso normal a termo) } \\
\text { apresentaram comprometimento global } \\
\text { nos domínios executivos, principal- } \\
\text { mente comportamentais. }\end{array}$ \\
\hline Anderson et al. ${ }^{17}$ & $\begin{array}{l}\text { Cognitive and Educational } \\
\text { deficits in children Born } \\
\text { extremely preterm }\end{array}$ & $\begin{array}{l}\text { Analisar o perfil das habilidades } \\
\text { cognitivas e comportamentais } \\
\text { em crianças nascidas abaixo de } \\
26 \text { semanas de IG. } \\
\text { Revisão de literatura }\end{array}$ & $\mathrm{IG}<26$ semanas. & $\begin{array}{l}\text { Crianças extremamente prematuras } \\
\text { apresentam altos índices de atraso de } \\
\text { desenvolvimento cognitivo e comporta- } \\
\text { mental que aumentam com a diminui- } \\
\text { ção da idade gestacional. }\end{array}$ \\
\hline $\begin{array}{l}\text { Woodward } \\
\text { et al. }{ }^{18}\end{array}$ & $\begin{array}{l}\text { Object working memory } \\
\text { deficits predicted by early } \\
\text { brain injury and develop- } \\
\text { ment in the preterm infant. }\end{array}$ & $\begin{array}{l}\text { Comparar o desempenho cog- } \\
\text { nitivo de crianças prematuras } \\
\text { e de termo aos dois anos de } \\
\text { idade. }\end{array}$ & $\begin{array}{l}\text { IG } 32 \text { semanas } \\
92 \text { crianças pré-termo e } \\
103 \text { a termo }\end{array}$ & $\begin{array}{l}\text { Crianças pré-termo possuem mais } \\
\text { dificuldade na memória de trabalho do } \\
\text { que as crianças a termo. }\end{array}$ \\
\hline $\begin{array}{l}\text { Zomignani } \\
\text { et al. }{ }^{19}\end{array}$ & $\begin{array}{l}\text { Desenvolvimento cerebral } \\
\text { em recém - nascidos } \\
\text { prematuros }\end{array}$ & $\begin{array}{l}\text { Descrever o perfil das altera- } \\
\text { çóes cognitivas e motoras de } \\
\text { crianças prematuras. Revisão de } \\
\text { literatura }\end{array}$ & Pré-termo & $\begin{array}{l}\text { Crianças nascidas prematuramente } \\
\text { apresentam alteraçóes cognitivas prin- } \\
\text { cipalmente nas habilidades viso-motor, } \\
\text { linguagem, atenção e comportamento. }\end{array}$ \\
\hline $\begin{array}{l}\text { Espírito Santo } \\
\text { et al. }{ }^{6}\end{array}$ & $\begin{array}{l}\text { Cognitive and behavioral } \\
\text { status of low birth weight } \\
\text { preterm children raised in } \\
\text { a developing country at } \\
\text { preschool age. }\end{array}$ & $\begin{array}{l}\text { Avaliar o desenvolvimento } \\
\text { cognitivo e comportamental em } \\
\text { idade pré-escolar de crianças } \\
\text { prematuras com baixo peso ao } \\
\text { nascimento }\end{array}$ & $\begin{array}{l}\mathrm{IG}<37 \text { semanas } \\
(80 \text { crianças pré-termo })\end{array}$ & $\begin{array}{l}\text { Crianças prematuras apresentaram me- } \\
\text { lhores resultados em testes relacionados } \\
\text { à capacidade de abstração e simboliza- } \\
\text { ção; os menores escores foram obtidos } \\
\text { em testes que avaliam a coordenação } \\
\text { visual motora, flexibilidade e velocida- } \\
\text { de de raciocínio. }\end{array}$ \\
\hline
\end{tabular}


Tabela 1

(Continuação)

\begin{tabular}{|c|c|c|c|c|}
\hline AUTOR & ARTIGO & OBJETIVOS & POPULAÇÁO & CONCLUSÁO \\
\hline Van Baar et al. ${ }^{20}$ & $\begin{array}{l}\text { Functioning at School Age } \\
\text { of Moderately Preterm } \\
\text { Children Born at } 32 \text { to } 36 \\
\text { Weeks' Gestational Age }\end{array}$ & $\begin{array}{l}\text { Descrever o perfil comporta- } \\
\text { mental de crianças nascidas pre- } \\
\text { maturas entre } 32 \text { a } 36 \text { semanas } \\
\text { de gestação }\end{array}$ & $\begin{array}{l}\text { IG entre } 32 \text { a } 36 \text { semanas } \\
377 \text { pré-termo e } 182 \text { a } \\
\text { termo }\end{array}$ & $\begin{array}{l}\text { Crianças prematuras apresentaram pro- } \\
\text { blemas de comportamento bem como } \\
\text { déficits de atenção e hiperatividade. }\end{array}$ \\
\hline $\begin{array}{l}\text { Aarnoudse-Moens } \\
\text { et al. }{ }^{21}\end{array}$ & $\begin{array}{l}\text { Meta-Analysis of Neuro- } \\
\text { behavioral Outcomes in } \\
\text { Very Preterm and/or Very } \\
\text { Low Birth Weight Children }\end{array}$ & $\begin{array}{l}\text { Analisar o comportamento e } \\
\text { funçấo executiva de crianças } \\
\text { nascidas prematuras e de muito } \\
\text { baixo peso. } \\
\text { Revisão de literatura }\end{array}$ & IG 33 semanas & $\begin{array}{l}\text { Problemas de atenção foram mais } \\
\text { pronunciados em prematuros e /ou } \\
\text { crianças de muito baixo peso. }\end{array}$ \\
\hline Rodrigues et al. ${ }^{9}$ & $\begin{array}{l}\text { Learning difficulties in } \\
\text { schoolchildren born with } \\
\text { very low birth weight }\end{array}$ & $\begin{array}{l}\text { Investigar a associação entre } \\
\text { crianças nascidas prematuras de } \\
\text { muito baixo peso e a dificulda- } \\
\text { de de aprendizagem na idade } \\
\text { escolar. } \\
\text { Revisão de literatura }\end{array}$ & Pré-termo & $\begin{array}{l}\text { Observou-se pior desempenho acadê- } \\
\text { mico de crianças prematuras quando } \\
\text { comparados aos nascidos a termo. O } \\
\text { risco de evoluir com dificuldades de } \\
\text { aprendizagem mostrou-se maior con- } \\
\text { forme menor o peso ao nascimento. }\end{array}$ \\
\hline Marlow et al. ${ }^{22}$ & $\begin{array}{l}\text { Motor and Executive Func- } \\
\text { tion at } 6 \text { Years of Age After } \\
\text { Extremely Preterm Birth }\end{array}$ & $\begin{array}{l}\text { Investigar a hipótese de que os } \\
\text { déficits de função neuromotora } \\
\text { e executiva podem contribuir } \\
\text { de forma independente ao } \\
\text { insucesso escolar. }\end{array}$ & IG 26 semanas & $\begin{array}{l}\text { A deficiência nas funções motoras, viso } \\
\text { espaciais e sensório-motoras, incluindo } \\
\text { planejamento, autocontrole, inibição e } \\
\text { persistência motora, contribui com as } \\
\text { dificuldades de compreensáo e cogni- } \\
\text { ção encontradas em crianças pré-termo, } \\
\text { e também pelo baixo desempenho } \\
\text { escolar dessas crianças aos } 6 \text { anos de } \\
\text { idade. }\end{array}$ \\
\hline Marlow et al..$^{23}$ & $\begin{array}{l}\text { Neurologic and develo- } \\
\text { pmental disability at six } \\
\text { years of age after extremely } \\
\text { preterm birth }\end{array}$ & $\begin{array}{l}\text { Investigar a alta prevalência } \\
\text { de problemas neurológicos e } \\
\text { deficiências de desenvolvimen- } \\
\text { to na criança durante os dois } \\
\text { primeiros anos de vida. }\end{array}$ & $\begin{array}{l}\text { IG } 26 \text { semanas } \\
241 \text { crianças no grupo } \\
\text { experimental e } 160 \\
\text { grupo controle }\end{array}$ & $\begin{array}{l}\text { Os testes cognitivos revelaram defici- } \\
\text { ência de } 21 \% \text { nas crianças prematuras, } \\
\text { quando comparadas a crianças a termo } \\
\text { o valor aumentou para } 41 \% \text { aos } 6 \text { anos } \\
\text { de idade. }\end{array}$ \\
\hline
\end{tabular}

brasileiras estão em crescimento, com diversos estudos já apresentados sobre a evolução das crianças pré-termo em idade escolar ${ }^{1-4,7,8,13,19,25-30,33,34,41}$.

Torna-se necessária a pesquisa e análise do perfil cognitivo e motor dessas crianças no nosso país devido à extensa populaçáo local encontrada, e a demanda de deficiências futuras observadas. Desse modo, o profissional da saúde (fisioterapeutas, educadores, médicos) pode oferecer um prognóstico bem como direcionar melhor suas condutas.

\section{CONCLUSÃO}

Concluímos que crianças nascidas prematuramente apresentam menores escores tanto nas tarefas cognitivas (dificuldade de memória e desatenção) quanto motoras (funçóes executivas e de habilidade) quando comparadas com crianças nascidas a termo.

Sugere-se que novos estudos nessa área sejam conduzidos, de forma que os pais, profissionais da área da saúde e educadores infantis, envolvidos com o pré-ter- mo, consigam avaliar e intervir nas necessidades de cada criança. A educação e divulgação sobre o tema devem ser realizadas, a fim de reduzir os transtornos de aprendizado que a criança vai apresentar, respeitando suas limitaçóes e minimizando consequentemente o insucesso escolar.

\section{REFERÊNCIAS}

1.Silveira MF, Santos IS, Barros AL, Matijasevich A, Barros FC, Victora CG. Aumento da prematuridade no Brasil: revisão de estudos de base populacional. Rev Saúde Públ 2008;42(5):957-964. http://dx.doi.org/10.1590/S0034-89102008000500023

2.Cascaes AM, Gauche H, Baramarchi FM, Borges CM, Peres KG. Prematuridade e fatores associados no estado de Santa Catarina, Brasil, no ano de 2005: analise dos dados do sistema de informaçôes sobre nascidos vivos. Cad Saúde Pública 2008;24(5):1024-1032.

http://dx.doi.org/10.1590/S0102-311X2008000500009

3.Bettiol H, Barbieri MA, Silva AAM. Epidemiologia do Nascimento pré-termo: tendências atuais. Rev. Bras de GinecolObstet 2010;32(2):57-60.

4.Kilsztajn S, Rossbach A, Carmo MSN, Sugahara GTL. Assistência pré-natal, baixo peso e prematuridade no estado de São Paulo, 2000. Rev Saúde Públ 
2003;37(3):303-310.

http://dx.doi.org/10.1590/S0034-89102003000300007

5.Ayres AM. Alteraçóes Cognitivas e Comportamentais Frequentes na Prematuridade. In: Pachi PR. O Pré-Termo Morbidade, Diagnóstico e Tratamento. Editora Roca, 2003, p.485-500.

6.Espírito Santo JL, Portuguez M W, Nunes ML. Cognitive and behavioral status of low birth weight preterm children raised in a developing country at preschool age. J Pediatric 2009;85(1):35-41.

http://dx.doi.org/10.1590/S0021-75572009000100007

7.Willrich A, Azevedo CCF, Fernandes JO. Desenvolvimento motor na infância: influência dos fatores de risco e programas de intervençấo. Rev Neurocienc 2008;17(1)51-56.

8.Bordim MBM, Linhares MBM, Jorge SM. Aspectos cognitivos e comportamentais na média meninice de crianças nascidas pré-termo e com muito baixo peso. PsicTeor e Pesq 2001;17;1:49-57.

9.Rodrigues MC, Mello RR, Fonseca SC. Learning difficulties in schoolchildren born with very low birth weight. J Pediatric 2006;82:6-14.

http://dx.doi.org/10.2223/JPED.1429

10.SM, Zheng H, Tang Y, Roth J. Early School-Age Outcomes of Late Preterm Infants. Pediatrics 2009;123;622-629.

http://dx.doi.org/10.1542/peds.2008-1405

11.Muthanna S, Marlow N, Wolke D, EPICure study Group. Pervasive Behavior problems at 6 years of age in a total population sample of children born at $\leq 25$ weeks of gestation. Pediatrics 2008;122:562-573.

http://dx.doi.org/10.1542/peds.2007-3231

12.Delobel-Ayoub M, Arnaud C, White-Koning M, Casper C, Pierrat V, Garel $\mathrm{M}$, et al. Behavioral Problems and Cognitive Performance at 5 Years of Age after Very Preterm Birth: The EPIPAGE Study. Pediatrics 2009;123;1485-1492. http://dx.doi.org/10.1542/peds.2008-1216

13.Magalhães LC, Catarina PW, Barbosa VM, Macini MC, Paixão ML. Estudo Comparativo sobre o desempenho perceptual e motor na idade escolar em crianças nascidas pré-termo e a termo. Arq Neuropsiquiatr 2003;61(2-A):250-255. http://dx.doi.org/10.1590/S0004-282X2003000200016

14. Hughes FLA. Motor, cognitive, and behavioral disorders in children born very preterm. Dev Med Child Neurol 2003;45:97-103.

http://dx.doi.org/10.1111/j.1469-8749.2003.tb00912.x

15.Sullivan MC, McGrath MM. Perinatal morbidity, mild motor delay, and later school outcomes. Dev Med Child Neurol 2003;45:104-112.

http://dx.doi.org/10.1111/j.1469-8749.2003.tb00913.x

http://dx.doi.org/10.1017/S0012162203000203

16.Anderson PJ, Doyle LW, and Victorian Infant Collaborative Study group. Executive Functioning in School-Aged Children Who Were Born Very Pretermor with Extremely Low Birth Weight in the 1990. Pediatrics 2004;114;50-57. http://dx.doi.org/10.1542/peds.114.1.50

17.Anderson PJ, Doyle LW. Cognitive and Educational Deficits in Children Born Extremely Preterm. Semin Perinatol 2008;32:51-58.

http://dx.doi.org/10.1053/j.semperi.2007.12.009

18. Woodward LJ, Edgin JO,Thompson D, Inder T. Object working memory deficits predicted by early brain injury and development in the preterm infant. Brain 2005;128:2578-2587.

http://dx.doi.org/10.1093/brain/awh618

19.Zomignani AP, Zambelli HJL, Antonio MA. Desenvolvimento cerebral em recém-nascidos prematuros. Rev Paul Pediatr 2009;27(2):198-203.

http://dx.doi.org/10.1590/S0103-05822009000200013

20.Van Baar AL, Vermaas J, Knots E, Kleine MJK and Soons P.Functioning at School Age of Moderately Preterm Children Born at 32 to 36 Weeks' Gestational Age. Pediatrics 2009;124:251-257.

http://dx.doi.org/10.1542/peds.2008-2315
21.Aarnoudse-Moens SCH, Weisglas- Kuperus N, Jaap Van JBG. Meta-Analysis of Neurobehavioral Outcomes in Very Preterm and/or Very Low Birth Weight Children. Pediatrics 2009;124:717-728.

http://dx.doi.org/10.1542/peds.2008-2816

22.Marlow N, Hennessy EM, Bracewell MA, Wolke D, EPICure Study Group. Motor and Executive Function at 6 Years of Age after Extremely Preterm Birth. Pediatrics 2007;120:793-804.

http://dx.doi.org/10.1542/peds.2007-0440

23. Marlow N, Wolke D, Bracewell MA, Muthanna S. The EPICure study Group. Neurologic and Developmental Disability at Six Years of Age after Extremely Preterm Birth. N Engl J Med 2005;352;1:9-19.

http://dx.doi.org/10.1056/NEJMoa041367

24.Chauhan SP, Scardo JA, Hayes E, Abuhamad AZ, Berghella.Twins: prevalence, problems, and preterm births. Am J Obstetrics 2010;volume:305-315. 25.Lucena NMG, Aragão POR, Andrade SMMS, Lucena LC, Melo LGB, Rocha TVR. Estudo do desenvolvimento motor primário de crianças em idade escolar submetidas à avaliação psicomotora. Arq Ciênc Saúde 2009;16(3):120126.

26.Bühler KEB, Flabiano FC, Mendes AE, Limongi SCO.Construção da permanência do objeto em crianças nascidas pré-termo muito baixo peso. Rev CEFAC 2007;9(3):3-7.

http://dx.doi.org/10.1590/S1516-18462007000300003

27. Oliveira LN, Lima MCMP, Gonçalves VMG. Acompanhamento de lactentes com baixo peso ao nascimento: aquisiçấo de linguagem. Arq Neuropsiquiatr 2003;61(3B):802-807.

http://dx.doi.org/10.1590/S0004-282X2003000500019

28. Rugolo LM. Crescimento e desenvolvimento a longo prazo do prematuro extremo. J Pediatric 2005;81(1 Supl):S101-S110.

29. Carvalho AVE, Linhares MBM, Martinez FE. História de Desenvolvimento e Comportamento de Crianças Nascidas Pré-termo e Baixo Peso (< $1.500 \mathrm{~g})$. PsicolReflCrít 2001;14(1):1-33.

30.Méio MDBB, Lopes CS, Morsch DS, Monteiro APG, Rocha SB, Borges RA, et al. Desenvolvimento cognitivo de crianças prematuras de muito baixo peso na idade pré-escolar. J Pediatric 2004;80:495-502.

http://dx.doi.org/10.1590/S0021-75572004000800012

31.Castro AG. Lima MC, Aquino RR, Eickmann SH. Sensory oral motor and global motor development of preterm infants. Pró-Fono RAtualCient 2007;19(1):29-38.

http://dx.doi.org/10.1590/S0104-56872007000100004

32.Cooke RWI. Are there critical for brain in children born preterm. Arch Dis Child Fetal Neonatal 2006;91:17-20.

http://dx.doi.org/10.1136/adc.2005.077438

33. Méio MDBB, Lopes CS, Morsch DS. Fatores Prognósticos para o desenvolvimento cognitivo de prematuros de muito baixo peso. Rev. Saúde Públ 2003;37(3):311-318.

http://dx.doi.org/10.1590/S0034-89102003000300008

34.Meio MDBB, Lopes CS, Sichieri R,Morsch DS. Confiabilidade do Teste WPPSI-R na avaliaçấo do desenvolvimento cognitivo de pré-escolares. Cad Saúde Pública 2001;17(1):99-105.

http://dx.doi.org/10.1590/S0102-311X2001000100010

35.Saigal S, Stoskopf B, Boyle M, Paneth N, Pinelli J, Streiner D, et al. Comparison of Current Health, Functional Limitations, and Health Care Use of Young Adults Who Were Born With Extremely Low Birth Weigh and Normal Birth Weight. Pediatrics 2007;119(3):e562-e573.

http://dx.doi.org/10.1542/peds.2006-2328

36.Saigal S, Stoskopf B, Streiner D, Boyle M, Pinelli J, Paneth N, et al. Transition of Extremely Low-Birth-Weight Infants from Adolescence to Young Adulthood Comparison with Normal Birth-Weight Controls. JAMA 
2006;295(6):667-675.

http://dx.doi.org/10.1001/jama.295.6.667

37.Bhutta AT, Cleves MA, Casey PH, Cradock MM, Anand KJ. Cognitive and behavioral outcomes of school-agd children who were born preterm: a meta-analysis. JAMA 2002;288:728-737.

http://dx.doi.org/10.1001/jama.288.6.728

38.Soria-Pastor S Padilla N, Zubiaurre-Elorza L, Ibarretxe-Bilbao N. Decreased Regional Brain Volume and Cognitive Impairment in Preterm Children at Low Risk. Pediatrics 2009;124;1161-1170.

http://dx.doi.org/10.1542/peds.2009-0244
39.Saigal S, Doyle LW. An overview of mortality and sequelae of preterm birth from infancy to adulthood. Series 2008;371(19):261-269.

40.Butcher PR, Van Braeckel K,Bouma A, Einspieler C, Stremmelaar EF, Bos AF. The quality of preterm infants' spontaneous movements: an early indicator of intelligence and behavior at school age. J Child Psychol Psychiatr 2009; 50:8:920-930.

http://dx.doi.org/10.1111/j.1469-7610.2009.02066.x

41.Pinto EB. O Desenvolvimento do Comportamento do Bebê Prematuro no Primeiro Ano de Vida. Psicol Refl Crít 2008;22(1):76-85.

http://dx.doi.org/10.1590/S0102-79722009000100011 